\title{
Sunflower Array Antenna with Adjustable Density Taper
}

\author{
Maria Carolina Viganó, ${ }^{1}$ Giovanni Toso, ${ }^{2}$ Gerard Caille, ${ }^{3}$ Cyril Mangenot, ${ }^{2}$ and Ioan E. Lager ${ }^{1}$ \\ ${ }^{1}$ International Research Centre for Telecommunications and Radar (IRCTR), Delft University of Technology, \\ Mekelweg 4, P.O. Box 5031, 2628 GA Delft, The Netherlands \\ ${ }^{2}$ European Space Agency, ESA-ESTEC, 2200 AG Noordwijk, The Netherlands \\ ${ }^{3}$ Thales Alenia Space, 26 Avenue J. F. Champollion, 31037 Toulouse, Cedex 1, France
}

Correspondence should be addressed to Maria Carolina Viganó, m.c.vigano@tudelft.nl

Received 24 September 2008; Accepted 5 January 2009

Recommended by Stefano Selleri

A deterministic procedure to design a nonperiodic planar array radiating a rotationally symmetric pencil beam pattern with an adjustable sidelobe level is proposed. The elements positions are derived by modifying the peculiar locations of the sunflower seeds in such a way that the corresponding spatial density fits a Taylor amplitude tapering law which guarantees the pattern requirements in terms of beamwidth and sidelobe level. Different configurations, based on a Voronoi cell spatial tessellation of the radiative aperture, are presented, having as a benchmark the requirements for a typical multibeam satellite antenna.

Copyright (C) 2009 Maria Carolina Viganó et al. This is an open access article distributed under the Creative Commons Attribution License, which permits unrestricted use, distribution, and reproduction in any medium, provided the original work is properly cited.

\section{Introduction}

Communication satellites use multiple beam antennas providing downlink and uplink coverages over a field of view for high data rate, multimedia, or mobile personal communication applications. High gain, multiple overlapping spot beams, using both frequency and polarization reuse, provide the needed coverage. In order to generate high gain spot beams, electrically large antenna apertures are required. These apertures may be generated by either reflectors or phased arrays. Phased arrays would be a natural choice to generate multiple beams but up to now the poor efficiency, the high cost, and the deployment complexity of active arrays have been their main drawbacks, limiting their use onboard satellites. These drawbacks are mainly due to the required distributed and tapered power amplification which is inducing poor power efficiency.

Aperiodic arrays with equiamplitude elements permit to mitigate these limitations and represent a valid alternative to traditional periodic phased arrays with amplitude tapering. Resorting to aperiodic arrays with equiamplitude fed elements is particularly effective for the design of large arrays working in transmission. This type of antenna architecture is considered extremely promising for achieving a multibeam coverage on the Earth from a geostationary satellite [1-4].
Unequally spaced arrays have several interesting characteristics and may offer some potential advantages with respect to periodic arrays [2]. Firstly, aperiodic arrays allow the reduction of the sidelobe level (SLL) without resorting to an amplitude tapering. A second useful property of aperiodic arrays is the possibility to reduce the number of elements in one assigned aperture without major impact on the beamwidth. The reduction in the number of elements, with respect to the corresponding periodic array, depends on the required aperture efficiency and on the field of view where the assigned sidelobe level is imposed (based on the desired scanning range and regulatory aspects). Thirdly, sparse arrays can effectively be employed for spreading out the energy that would otherwise accumulate in grating lobes (GL) due to the wide interelement spacing.

In terms of limitations, nonperiodic arrays exhibit a reduced aperture efficiency when identical, non-equispaced elements are used. As a consequence, a reduced maximum equivalent isotropically radiated power (EIRP) is obtained if not compensated by an increase of the power radiated by each active chain. Furthermore, implementation constraints, as a nonregular lattice, may jeopardize the use of generic building blocks, with consequences on the costs. This particular drawback may be mitigated by implementing a set of different types of subarrays to fill the whole aperture. 
Up to now, sparse and thinned arrays have been rarely used, essentially because of the complexity of their analysis and synthesis with a reduced knowledge, as a consequence of their radiative properties. The main concern in the design of sparse arrays is to find an optimal set of element spacing to meet the array specifications, while assuming a uniform excitation for practical convenience.

The synthesis of aperiodic arrays is a known problem in the antenna community [5-22]. It is interesting to observe that while in the 1960s and 1970s mainly deterministic solutions have been proposed, in the last years procedures based on statistic global optimization techniques have been mainly presented. Recently, in [22], the simple and elegant spatial tapering deterministic solution introduced in the papers of Doyle [11] and Skolnik [17], and qualitatively in the work of Willey [7], has been revisited and improved. Deterministic solutions present two important advantages with respect to statistical algorithms: they allow obtaining results in real time and offer a solution with a controllable accuracy. Moreover, the results obtained applying deterministic solutions may be directly used or adopted as starting point for a further optimization based on a numerical technique which can take into account other constraints (like maximum and minimum spacing between the radiators, etc.).

The problem of aperiodic arrays has recently gained a renewed interest especially for the design of multibeam satellite antennas [1-4]. Most of the techniques presented for the design of aperiodic arrays deal with the case of linear arrays. When approaching the planar case, the designer has a higher number of degrees of freedom but the problem increases also in complexity. In some papers, an aperiodic planar lattice organized in rings has been proposed [1214]. Arrays organized in rings permit having a pattern with good symmetry properties, allow the reduction and control of GL, and their design is simplified because the elements positions can be expressed as the product of two functions one controlling the angular position of the elements and one controlling the distance of the rings from the center.

In this paper, the equiamplitude elements constituting the aperiodic array are placed on a lattice reproducing the positions of the sunflower seeds, opportunely adjusted according to a desired amplitude tapering. This type of lattice is selected essentially because it guarantees a really good radial and azimuthal spreading in the element positions. As a consequence, the pattern in the sidelobes and grating lobes region tends having a plateau-like shape $[9,16]$, avoiding the presence of high narrow peaks. Moreover, by adjusting the element positions using a simple parametric equation, a beamwidth can be selected and the SLL kept under an assigned value.

An aperiodic planar array with the elements organized according to a sunflower lattice has been already proposed in [23]. However, the spatial density of the elements in [23] is uniform. As a consequence, since the elements are equiamplitude, the equivalent amplitude tapering is uniform as well so that this type of array guarantees only a good suppression of the GL, without the ability of controlling the SLL.
The hereby proposed sunflower lattice is completely adjustable in order to follow stringent requirements on the beamwidth and the SLL without using any amplitude taper. This planar array can be considered in the design of a transmitting direct radiating array for a satellite communication antenna on a geostationary satellite.

The paper now proceeds as follows. In Section 2 the radiation pattern is introduced for a generic array, in Section 3 the definition of the element density function is given and discussed for the uniform case. In Section 4 a procedure to adjust the spatial density according to a Taylor amplitude tapering is presented. In Section 5 the requirements of a typical telecommunication multibeam antenna are introduced. Finally, in Section 6 some numerical results, in order to test the functionality of the proposed configuration, are presented. Additionally, the appendixes provide more general information on spirals and discuss the normalized element density that is used in the course of the element placement.

\section{Array Radiation Pattern}

The antenna radiation pattern of a planar array is given by

$$
\begin{aligned}
E(\theta, \phi)=\sum_{n=1}^{N} a_{n} F_{n} \exp (j k & {\left[x_{n} \sin (\theta) \cos (\phi)\right.} \\
& \left.\left.+y_{n} \sin (\theta) \sin (\phi)\right]\right),
\end{aligned}
$$

where $N$ is the total number of elements, $a_{n}$ represents the excitation coefficient for the $n$th element and $F_{n}$ its radiation pattern, $x_{n}$ and $y_{n}$ are the $n$th element positions in the $x O y$ plane, $\theta$ is the elevation angle measured from the $O z$ axis, and $\phi$ is the azimuth angle measured in the $x O y$ plane with respect to the $O x$ axis. Because the variables to be derived $\left(x_{n}\right.$ and $\left.y_{n}\right)$ appear inside an exponential function, the optimization problem is not linear. Moreover, $F_{n}$, unlike in periodic arrays, can change from element to element if a requirement on the minimum aperture efficiency (hence on the minimum gain) is enforced.

In the following section, a particular spiral configuration will be introduced as a starting point before the space taper is applied.

\section{Spiral Array with Uniform Spatial Density}

A well-known spiral is the Fermat one (see Figure 12) which has the property of enclosing equal areas within each turn. This spiral is often found in nature, as indicated in the appendix. The elements are placed along this spiral according to the following equations:

$$
\begin{array}{ll}
\rho_{n}=s \sqrt{\frac{n}{\pi}}, & \text { for } n=1, \ldots, N+1, \\
\phi_{n}=2 \pi n \beta_{1}, & \text { for } n=1, \ldots, N+1,
\end{array}
$$

where $\rho_{n}$ is the distance from the spiral center to the $n$th element, the parameter $\beta_{1}$ controls the angular displacement $\phi$ between two consecutive elements, and the parameter $s$ 
denotes the distance between the elements in the $x O y$ plane. Assume a sparse array deployed on a circular aperture of radius $R_{\text {ap }}$ along the Fermat spiral, with element locations given by (2) and (3). Note that $\rho_{N}$ is taken to correspond to $R_{\text {ap }}$, whereas $\rho_{N+1}$ is a virtual element places outside the aperture, its use is becoming obvious in what follows.

Let us now introduce a normalized element density function:

$$
\tilde{d}\left(\rho_{n}\right)=\frac{\left(R_{n}^{2}-R_{n-1}^{2}\right)_{\min }}{\left(R_{n}^{2}-R_{n-1}^{2}\right)}, \quad \text { for } n=1, \ldots, N,
$$

where $R_{n-1}$ and $R_{n}$ are the inner and outer radii of the annular rings enclosing the $n$th element, respectively, with $R_{0}$ being always taken as zero (see the appendix) for a justification for this choice of defining the normalized density function). Here, a choice is made to take the radii $R_{n}$ as

$$
R_{n}^{2}=\frac{\rho_{n+1}^{2}+\rho_{n}^{2}}{2} \text { for } n=1, \ldots, N .
$$

As recognizable from (4), the normalized density function corresponds to the current of a single element divided by the area of the relevant annular ring.

The lattice in [23] (see Figure 1) is characterized by a uniform density. On account of (5) and (2), it can be easily shown that

$$
\tilde{d}\left(\rho_{n}\right)=\frac{2\left(R_{n}^{2}-R_{n-1}^{2}\right)_{\min }}{\left(\rho_{n+1}^{2}-\rho_{n-1}^{2}\right)}=\frac{\pi R_{\mathrm{ap}}^{2}}{N s^{2}}=1 .
$$

This property is attractive when the interest focuses on avoiding GL only, without a control of the SLL. As for the SLL, it remains around $17 \mathrm{~dB}$, irrespective of the number of elements in the array and the spacing factor $s$. This is consistent with the element distribution replicating a uniform current distribution on a circular aperture.

It is now clear that the only possibility to control the SLL as well is by introducing a density taper. In the following section, it will be demonstrated how, by translating a Taylor amplitude tapering law [24] into a corresponding spatial density law, the SLL can be drastically reduced.

\section{Spiral Array with Density Tapering}

The spiral aperiodic lattice with a uniform element density introduced in the previous section is an excellent starting point to apply a space tapering process. The spreading of the elements in the spiral arms guarantees an optimal behavior in terms of GL even when the interelement spacing is larger than $\lambda$. In order to be able to control the SLL, it is possible to vary the elements positions with respect to the array center, thus obtaining an effect similar to an amplitude taper.

The space taper technique presented here consists of choosing a reference amplitude distribution whose pattern satisfies the assigned requirements and emulates it by varying the radiator distance from the center. Concretely, a Taylor amplitude taper law with a certain SLL and $\bar{n}$ [24] is selected as a reference. The locations of the elements in the sparse

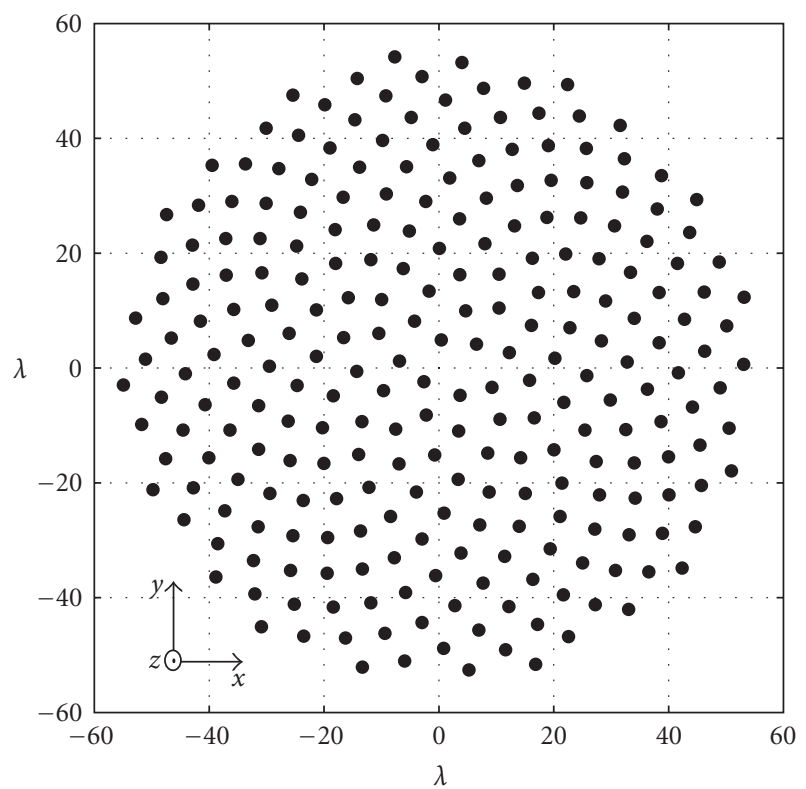

FIgURE 1: Distribution of the 250 elements in the uniform sunflower array antenna, as reported in [23].

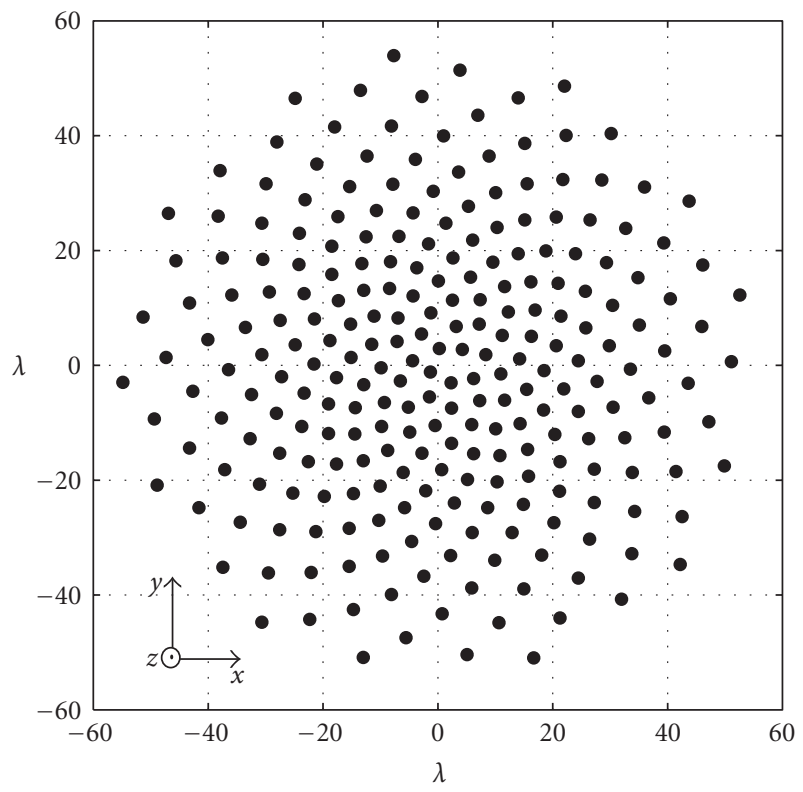

FIGURE 2: Distribution of the 250 elements in the tapered sunflower array antenna.

array are determined by means of a simple, 2 step algorithm: firstly $N$ circles of increasing radii $\rho_{n}, n=1,2, \ldots, N$ are selected by sequentially applying the relations

$$
\begin{aligned}
& 2 \pi \int_{R_{n-1}}^{\rho_{n}} A(r) r \mathrm{~d} r=\frac{2 \pi}{2 N} \int_{0}^{R_{\mathrm{ap}}} A(r) r \mathrm{~d} r, \\
& 2 \pi \int_{R_{n-1}}^{R_{n}} A(r) r \mathrm{~d} r=\frac{2 \pi}{N} \int_{0}^{R_{\mathrm{ap}}} A(r) r \mathrm{~d} r,
\end{aligned}
$$

starting from $R_{0}$ that is taken to be 0 . Here, $A(r)$ denotes the Taylor amplitude taper and $R_{\mathrm{ap}}$ is the radius of the complete 


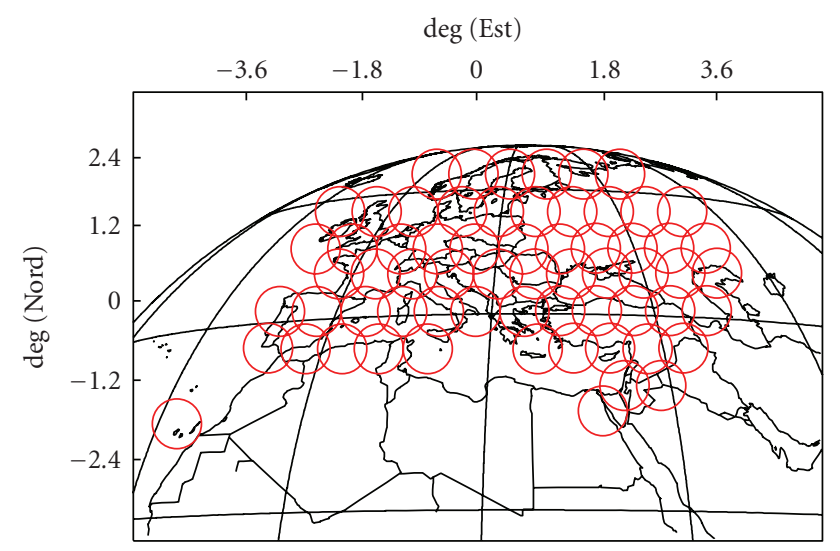

Figure 3: European multibeam coverage in a $1: 4$ frequency re-use scheme from a geostationary satellite.

circular aperture. Note that (7) emulates the desired taper by equating the surface integral over the annular ring delimited by $R_{n-1}$ and $\rho_{n}$ to half of the $N$ th part of the total aperture excitation. Subsequently, the element positions are determined by choosing their pertaining azimuth angle $\phi_{n}$ according to (3). The result of this placement strategy is illustrated in Figure 2 where a $56 \lambda$ aperture is filled with 250 elements distributed in a manner such to obtain a pattern similar to the one achievable with a Taylor amplitude law characterized by SLL $=32 \mathrm{~dB}$ and $\bar{n}=4$. A total number $N=250$ is selected as a good compromise between the performance in scanning and the cost. The choice for these values will be clearer in the following sections.

\section{Typical Requirements for a Multibeam Satellite Application}

The transmitting antenna considered in this study is operating in Ka-band (19.7-20.2 GHz) and may have a maximum diameter of $1.3 \mathrm{~m}$. The starting point considers the circular direct radiating array with dimensions deemed as sufficient to provide the required maximum gain and beamwidth. The array must generate 64 spot beams. The total frequency band is divided into 4 subbands, and each of them being assigned to a set of beams so that there are no adjacent pencil beams using the same resource. Figure 3 shows the footprint on the Earth of the 64 pencil beams.

In the last 3 rows of Table 1, the maximum sidelobe level in three different regions has been specified. The value in $\mathrm{dBi}$ has been preferred to the $\mathrm{dB}$ one as the configurations analyzed in Section 5 have different maximum directivity values.

In Figure 4, the array factor of the configurations presented in Figures 1 and 2, respectively, is plotted for two different $\phi$ cuts. In both uniform and tapered sunflower configurations, the array factor is remarkably stable in $\phi$, resulting in the area of interest, in practically rotationally symmetric radiation patterns. The array factor in Figure 4(b) is following the expected behavior until a certain $\theta$ angle at which the effects of the first pseudograting lobe (the

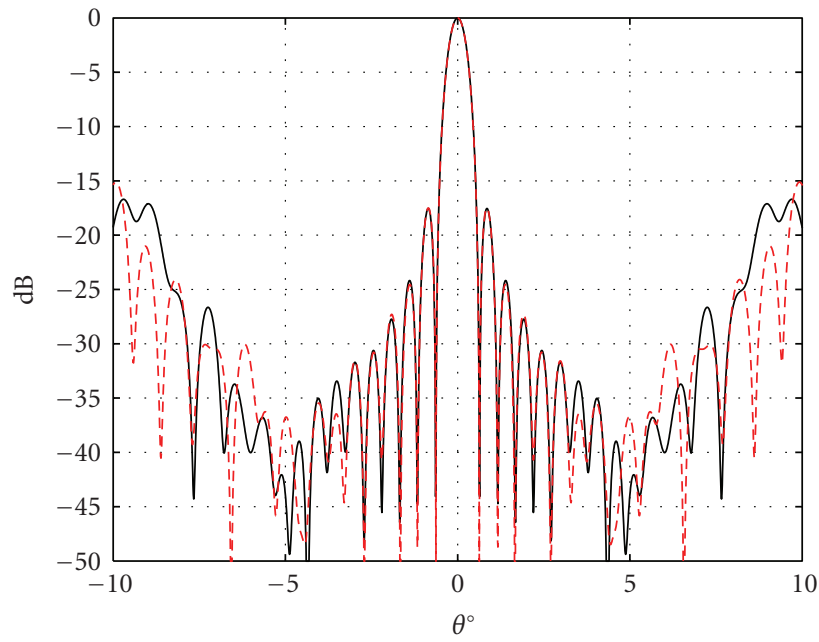

(a)

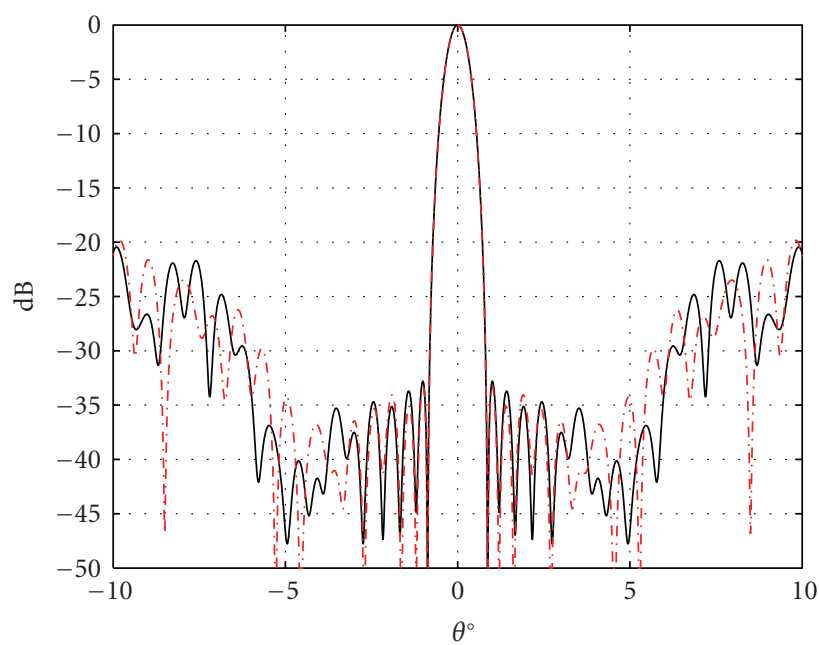

(b)

Figure 4: Array Factor, two different $\phi$ cuts $\left(\phi=0^{\circ}\right.$ in red dotted line and $\phi=90^{\circ}$ in black), for the array configuration in Figures 1 and 2 , respectively.

TABle 1: Mission requirements.

\begin{tabular}{lc}
\hline Number of spots & 64 \\
Spot diameter & $0.65^{\circ}$ \\
Inter-spot distance & $0.56^{\circ}$ \\
Rx band & $29.5-30.0 \mathrm{GHz}$ \\
Tx band & $19.7-20.2 \mathrm{GHz}$ \\
Frequency reuse & $1: 4$ \\
EOC gain & $43.8 \mathrm{dBi}$ \\
SLL in the first $\mp 4^{\circ}$ & $20 \mathrm{dBi}$ \\
SLL in the first $\mp 8^{\circ}$ & $25 \mathrm{dBi}$ \\
SLL in the first $\mp 90^{\circ}$ & $30 \mathrm{dBi}$ \\
\hline
\end{tabular}

energy of which being spread over a wider $\theta$ interval due to the nonperiodic placement) become visible. Considering the previous example in which the maximum interelement 


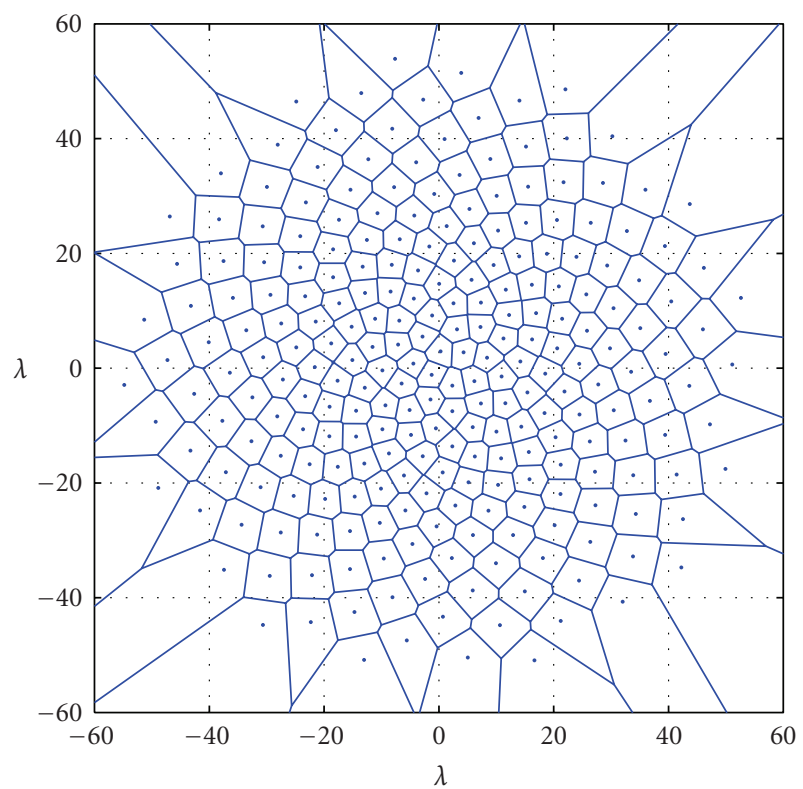

Figure 5: The Voronoi tessellation consisting of the cells enclosing the chosen phase centers.

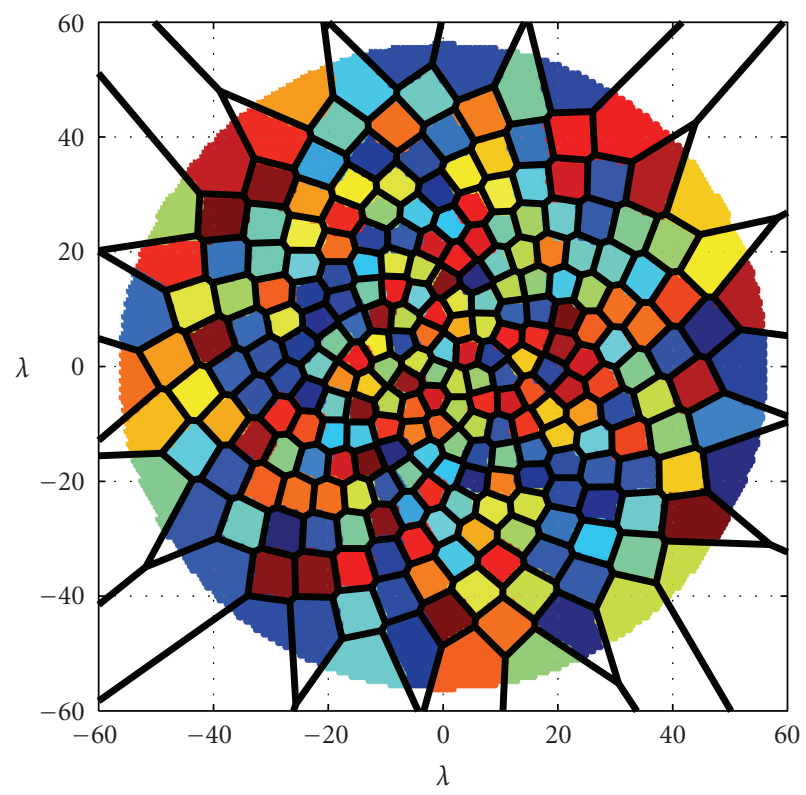

FIGURE 6: Subarray allocation and aperture subdivision corresponding to the Voronoi tessellation in Figure 5.

distance $D_{\max }=8.43 \lambda$, the first contribution of the grating lobe is expected at

$$
\theta_{\mathrm{GL}}=\sin ^{-1}\left(\frac{1}{D_{\max }}\right)=6.81^{\circ}
$$

In fact, in Figure 4(b), the pattern starts exceeding the imposed SLL around this $\theta_{\mathrm{GL}}$.

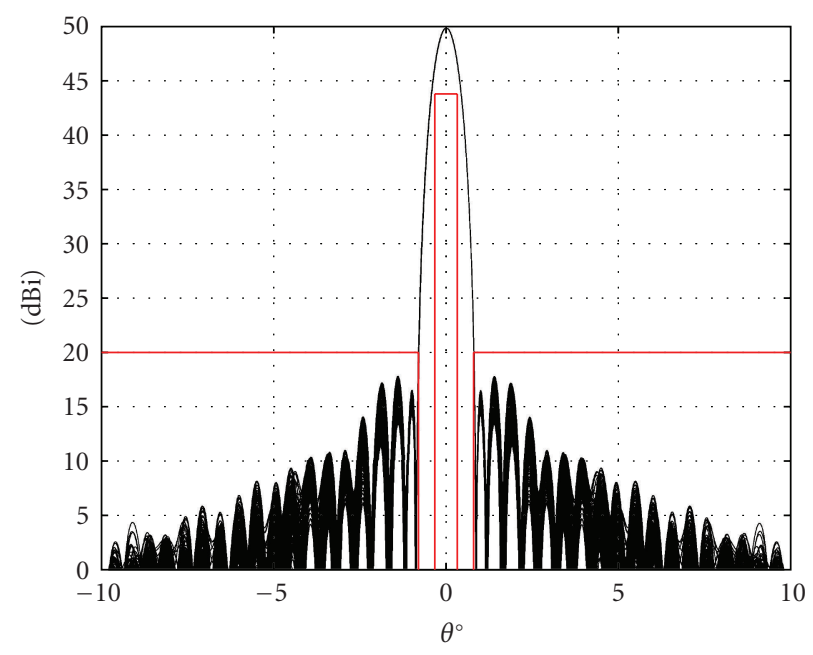

FIgURE 7: Array pattern for the configuration depicted in Figure 6. The beam is scanned to boresight. The red line corresponds to the requirement mask.

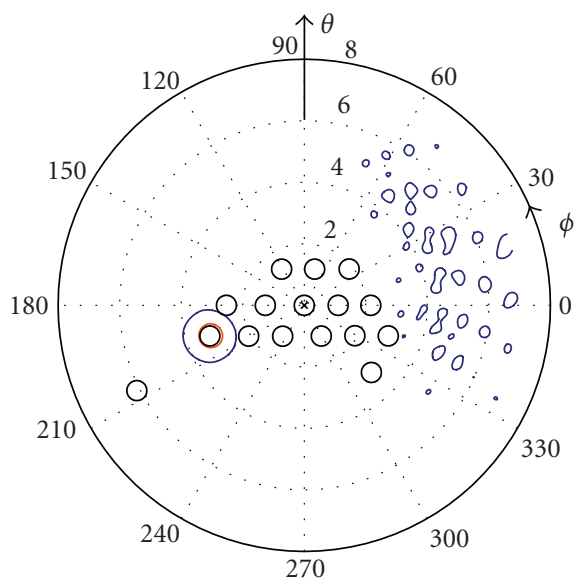

Figure 8: Array pattern for the configuration depicted in Figure 6. The beam is pointing at Europe edges. The red line represents the iso-level curves at $43.8 \mathrm{dBi}$, and the blue ones the iso-level curves at $20 \mathrm{dBi}$. The black circles represent the interfering area.

\section{Validation of the Technique}

The locations provided by the space taper process (see Figure 2) have been used as phase centers of the radiators in a planar array. Two different techniques are used to select the radiators.

6.1. First Approach: Using the Entire Aperture. The circular aperture with a maximum radius of $56 \lambda$ is completely filled with patches disposed on a regular lattice. The triangular grid is chosen because of its better performances compared to the regular rectangular one. The analytical equations in [25] are used to express the field of the elementary patch antenna, radiating on a ground plane, with side lengths equaling $0.42 \lambda$ and a rectangular cell surrounding it with side lengths $0.8 \lambda \times 0.85 \lambda$. In order to cover the complete 


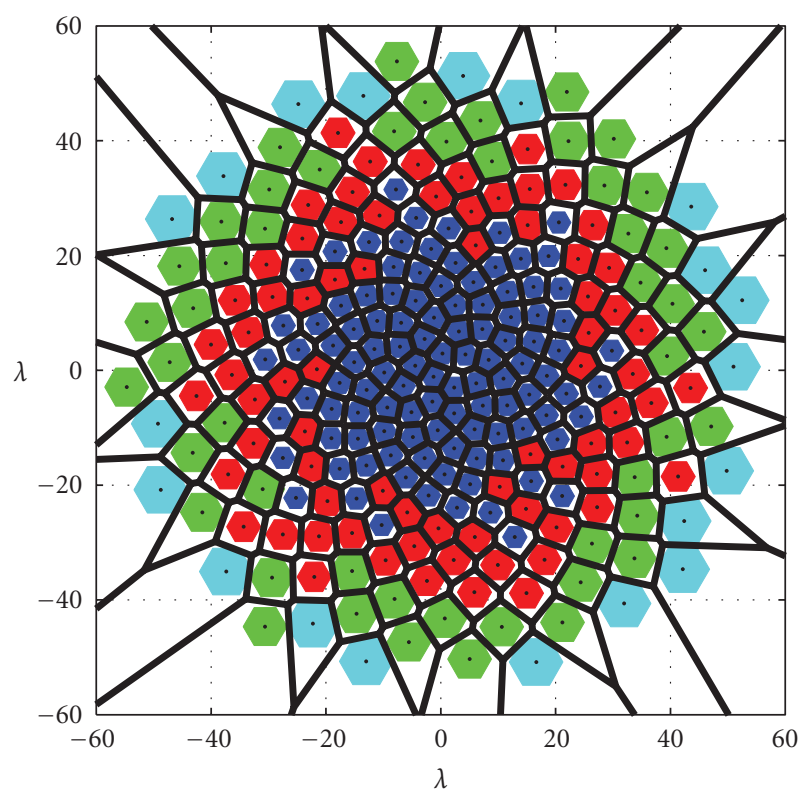

Figure 9: Hexagonal subarray positions and dimension after postprocessing.

aperture, with this patch choice, more than 15 thousands patches are required. These patches are then collected into subarrays.

The positions of the phase centers of the subarrays for $N=250$ are derived with the formulation presented in the previous section following a Taylor taper with SLL $=32 \mathrm{~dB}$ and $\bar{n}=4$. The subarray positions are then superimposed on the uniform array, as indicated in Figure 6. Each patch center is assigned to the closest subarray center that can be interpreted as assigning the relevant patch to the Voronoi cell [26] corresponding to the computed phase center. Note that the Voroni surface division (see Figure 5) provides an optimum tessellation of the available real estate.

To obtain the total radiation pattern, each radiation pattern of the subarray $F_{n}$ has been calculated and multiplied by the exponential that takes into account the positions of the phase center of that subarray given in (1).

Since the Voronoi cell shapes are close to circular ones, the subarray patterns result to be almost rotationally symmetric. This is an important property when the beam is scanned.

In Figure 7, the pattern for the beam pointing at boresight is plotted for $360 \phi$ cuts (one at every degree). The red line in this figure indicates the mask requirements given in Table 1. Figure 8 represents the radiation pattern when the beam is pointed at the Europe's edges: the blue and red lines are iso-level curves at $20 \mathrm{dBi}$ and $43.8 \mathrm{dBi}$, respectively, while the black circles enclose the regions of the coverage in which the same resource is used. As it can be seen from Figure 8, even when the beam is pointed at the Europe's edges, the pattern remains complaint with the requirements given in [1].

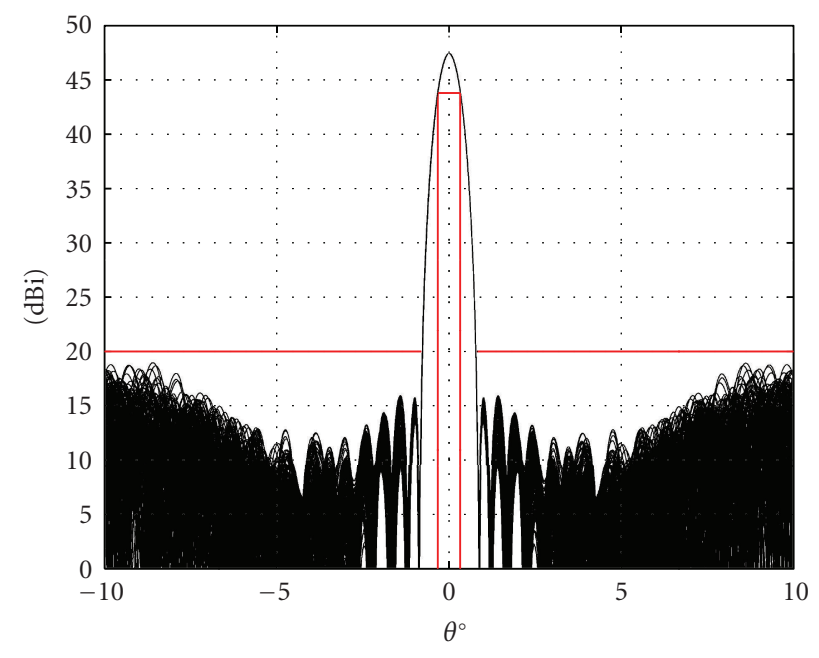

FIGURE 10: Array pattern for the configuration depicted in Figure 9. The beam is scanned to boresight. The red line corresponds to the requirement mask.

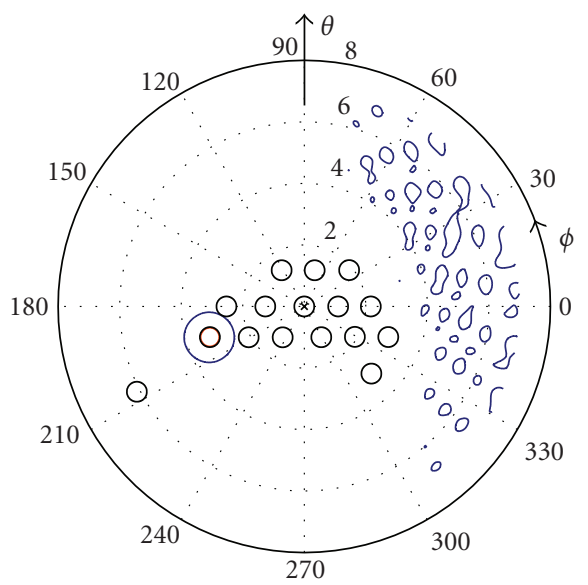

FIGURE 11: Array pattern for the configuration depicted in Figure 9. The beam is pointing at Europe edges. The red line represents the iso-level curves at $43.8 \mathrm{dBi}$, and the blue ones the iso-level curves at $20 \mathrm{dBi}$. The black circles represent the interfering area.

With this method, the entire surface available is used maintaining at the same time a very small number of controls (one for each subarray).

6.2. Second Approach: 4 Different Types of Subarrays. In this case, a more technology-oriented approach is considered: the aperture is filled as much as possible with predefined hexagonal subarrays. A limited number of these subarrays is selected as a compromise in order to keep the complexity and the cost limited while offering good performances. Four subarrays with different sizes have been selected and used to fill the array aperture. All the subarrays have a hexagonal shape and consist of 2, 3, 4, or 5 rings of elements surrounding the central one on a regular triangular lattice. The patches used in the subarrays are the same as the ones described in the previous subsection. 


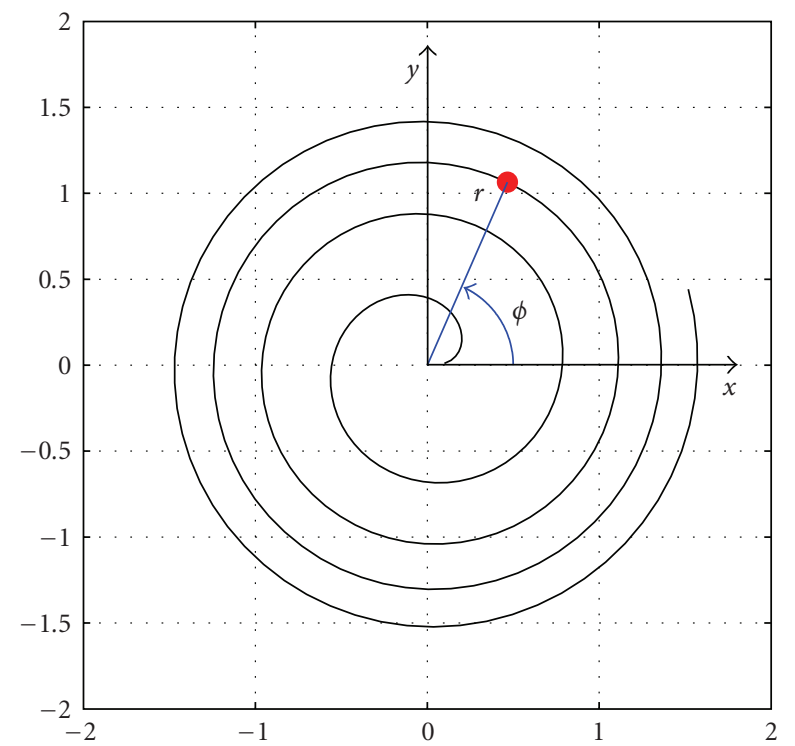

FIGURE 12: The Fermat spiral and its associated coordinate system.

The procedure consists of computing for each cell the radius of the maximum circle that can be inscribed in it. According to this value, the best hexagonal sub-array among the four available is selected and placed in the cell (see Figure 9). After this first step, a postprocessing is carried out in which every subarray is replaced by its larger version if no overlapping with its neighbor occurs. In the following case, 117 of the 250 subarrays were substituted during the postprocessing and this is the reason why the subarray placement in the array is not clearly divided into annular areas around the array center enclosing the same type of subarrays. The postprocessing allows increasing the aperture filling from $44.4 \%$ up to $60.9 \%$.

In Figure 9, the subarrays have been plotted in different colors depending on their size. Since the element placement is more dense in the center, smaller subarray dimensions are needed in the array middle while at the periphery larger hexagonal subarray can be accommodated improving the directivity but, depending on the maximum dimension, allowing for a limited beam scan only.

With the first approach, the results were exceeding the requirements but the physical implementation of the array would be too demanding since every subarray is different and has to be designed and tested individually. With the second approach proposed here, only 4 subarrays need to be generated and moreover, the feeding network will be easier to implement. The boresight radiation pattern for this configuration is depicted in Figure 10. As it can be noticed, the SLL is still within the specifications; the maximum gain drops by approximately $2 \mathrm{~dB}$ but the EOC gain is enforced. As a drawback, the array performance is optimal only when the beam is pointing at boresight. When the beam is scanned to the Europe edges, it is possible to maintain the SLL under the prescribed value in the area of interest but the requirement on the EOC gain cannot be reached as shown in Figure 11.

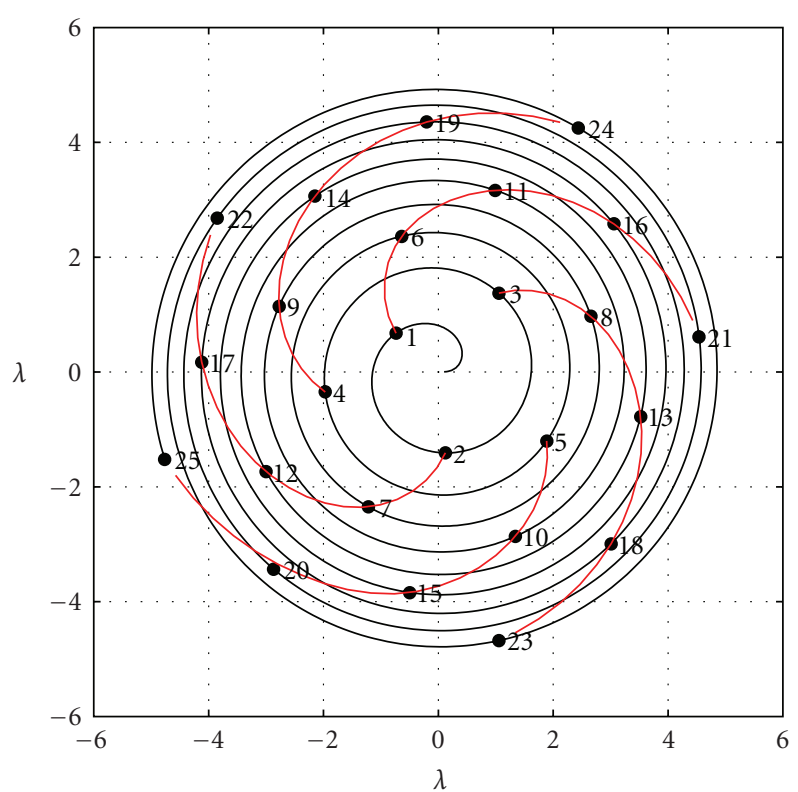

(a)

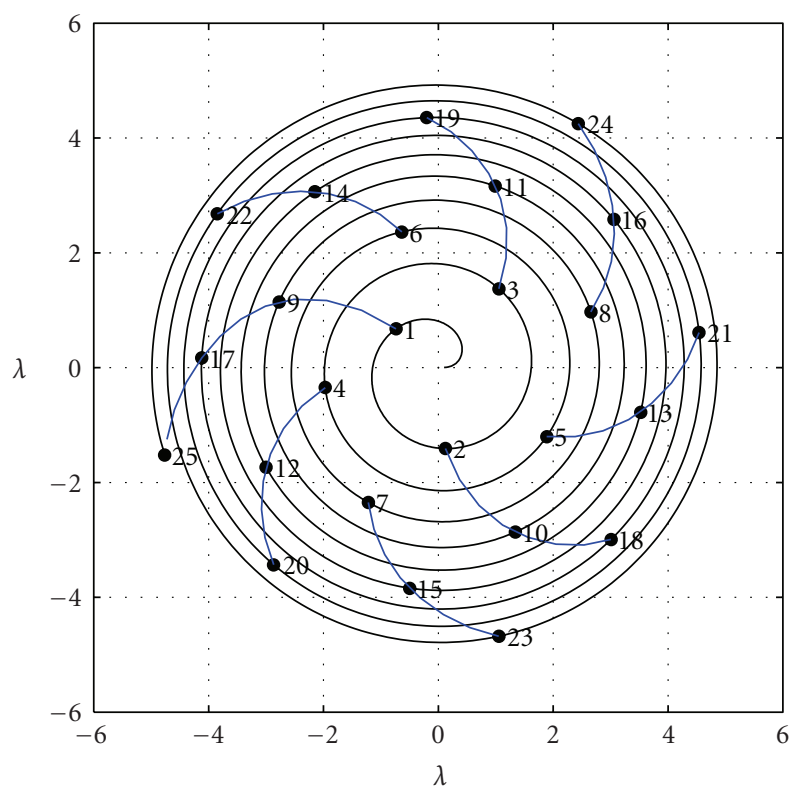

(b)

Figure 13: Sunflower array configuration: the elements are numbered starting from the center. In this case, 5 clockwise Fibonacci spirals appear, red line, and 8 anticlockwise, in blue line. The interval of identificative numbers between elements on the same Fibonacci spiral is always equal to the number of spirals occurring, in this figure notice $22-14=14-6=8$ and $24-19=19-14=5$.

\section{Conclusions}

A deterministic procedure to design aperiodic planar arrays which guarantees the control of SLL, GL, and beamwidth without using any amplitude tapering has been introduced. Starting from an array characterized by a uniform spatial density of the elements, the density function has been 
modified in order to fit a reference amplitude tapering. The design technique has been applied for the preliminary design of a Direct Radiating Array for a multibeam satellite communication mission.

\section{Appendices}

\section{A. Fibonacci Spirals}

Spirals are one of the most common regular shapes in nature: from the snail shell to the sunflower seed placement, to the Milky way arms. Different kinds of spirals are known in literature. Using a spiral placement for the elements of a planar array guarantees a good spreading of the energy associated to the side and grating lobes. Furthermore, a spiral lattice permits obtaining a quite uniform filling of a given aperture compared to other planar lattices like the ones organized in rings. A well-known spiral is the Fermat spiral (Figure 12) which has the property of enclosing equal areas within every turn. Its equation can be expressed in polar coordinates as

$$
\rho=a \sqrt{\phi}
$$

where $\rho$ is the distance from the spiral center, and $\phi$ is the angle that identifies the point position respect to the $x$ axis; the parameter a controls the distance between the spiral turns.

This spiral is quite often found in nature. In particular, there are leafs and seeds whose positions can be obtained by sampling a Fermat spiral equation, that is,

$$
\begin{aligned}
& \rho=\sqrt{n} b, \\
& \phi=\frac{2 \pi n}{c},
\end{aligned}
$$

and when it is important having a uniform subdivision of the space the parameters $b$ and $c$ are closely related to the Golden Ratio, also known as Fibonacci number since it represents the solution of the Fibonacci quadratic equation. For instance, the leaves around a stem use this positioning to share in an optimal way the space and the light [27].

The Fibonacci sequence is known since 1202 d.C., thanks to Leonardo son of Bonaccio from Pisa and his book Liber Abaci. This sequence has been widely analyzed and applied in different fields: from the description of particular plants to computer science, from crystallography to electrical engineering. By solving the Fibonacci quadratic equation [28]:

$$
\beta^{2} A_{n}=\beta A_{n}+A_{n}
$$

the following two roots are obtained:

$$
\begin{aligned}
& \beta_{1}=\frac{\sqrt{5}+1}{2}=\tau, \\
& \beta_{2}=\frac{1-\sqrt{5}}{2}=-\frac{1}{\tau} .
\end{aligned}
$$

In most of the applications, the first value has been used, but to characterize the spiral, both of them are usable. The divergence angle, also referred to as the golden angle, is defined as

$$
\text { golden angle }=\frac{360^{\circ}}{\beta_{1}^{2}}=360^{\circ}-\frac{360^{\circ}}{\beta_{2}^{2}} .
$$

Because this value is irrational, it is impossible to have two or more elements in the spiral array characterized by the same $\phi$ angle. The element packing results to be efficient. Interesting Fermat spirals could be also the ones with other irrational coefficients like $\sqrt{2}$. In the patent [23], $\beta_{1}$ is used for the element disposition along the spiral according to the formulation presented in (2) and (3). As it can be easily noticed, the positions of the elements in the sunflower array depend only on $n$ via a trivial equation.

The second type of spirals employed in this study is the Fibonacci one, namely, a particular kind of logarithmic spiral, where the ratio between radii evaluated at each $90^{\circ}$ is related to the golden ratio number. It is interesting to note that in a sunflower array configuration, when the elements are placed on a Fermat spiral at every $\beta_{1}$ degrees, the elements form sets of clockwise and anticlockwise Fibonacci spirals. The number of spirals in each set are two consecutive terms of the Fibonacci series as it can be seen in Figures 13(a) and 13(b). Another particular characteristic of this configuration is that in order to obtain for example the 5 clockwise spirals, it is sufficient to connect the elements on the Fermat spiral whose numbers difference is exactly 5 .

\section{B. Normalized Element Density Function}

Assume the case when a continuous, strictly positive, rotationally symmetric, normalized current amplitude distribution $A(r)$ on a circular aperture of radius $R_{\mathrm{ap}}$ needs to be mimicked by means of $N$ equiamplitude elements located at monotonically increasing distances $\rho_{n}, n=1, \ldots, N$. (All distances $r$ or radii $R$ employed in this appendix are considered with respect to the aperture center.) Let $R_{n}(n=$ $0, \ldots, N)$ be $N+1$ radii chosen such that $R_{n-1}<\rho_{n}<R_{n}$ for $n=1, \ldots, N$, with $R_{0}$ being taken to be zero. Note that for reasons that will be elaborated upon later, $R_{N}$ may be, and in most cases is, taken to be (slightly) larger than $R_{\mathrm{ap}}$. The monotonic increasing of the values $\rho_{n}$ combined with the choice for the radii $R_{n}$ ensures that inside in each annular ring of inner radius $R_{n-1}$ and outer radius $R_{n}$ there is only one radiator. With these prerequisites, an equivalent discrete amplitude density function can be defined by means of the expression

$$
d\left(\rho_{n}\right)=\frac{K}{\pi\left(R_{n}^{2}-R_{n-1}^{2}\right)}, \quad \text { for } n=1, \ldots, N,
$$

where the $K$ denotes the constant excitation of each of the $N$ elements. In view of ensuring the consistency of this definition, the radii $R_{n-1}$ and $R_{n}$ are chosen such that

$$
\int_{R_{n-1}}^{R_{n}} A(r) r \mathrm{~d} r=K, \quad \text { for } n=1, \ldots, N .
$$


Furthermore, in view of ensuring identical aggregate excitation over the aperture, the constant $K$ is adopted as

$$
K=\frac{1}{N} \int_{0}^{R_{\text {ap }}} A(r) r \mathrm{~d} r
$$

By now invoking the mean function theorem, the area of the annular ring in the denominator of (B.1) can be rewritten as

$$
\begin{aligned}
\pi\left(R_{n}^{2}-R_{n-1}^{2}\right) & =\frac{1}{A}\left(\xi_{n}\right) \int_{R_{n-1}}^{R_{n}} A(r) r \mathrm{~d} r \\
& =\frac{K}{A}\left(\xi_{n}\right), \quad \text { for } n=1, \ldots, N,
\end{aligned}
$$

with $\xi_{n}(n=1, \ldots, N)$ being an unspecified point in the intervals $\left[R_{n-1}, R_{n}\right]$. Substituting (B.4) in (B.1) then yields

$$
d\left(\rho_{n}\right)=A\left(\xi_{n}\right), \quad \text { for } n=1, \ldots, N .
$$

Due to the choice for $\rho_{n}$ and $\xi_{n}$, the distance $\left|\rho_{n}-\xi_{n}\right|$ is bounded above by $R_{n}-R_{n-1}$, which, in view of complying to (B.3) and of the continuity of $A(\rho)$, becomes arbitrarily small for arbitrarily large $N$-s. Consequently, the (discrete) amplitude density function can be made to approximate with arbitrary accuracy of the original current density.

In order to prevent possible above unit values of the discrete amplitude density $d\left(\rho_{n}\right)$ that may occur in the case when $N$ is small, this quantity is now normalized to its largest value. By accounting for the fact that $\left.\max \left[d\left(\rho_{n}\right)\right]\right|_{n=1, \ldots, N}$ corresponds to the minimum $R_{n}^{2}-R_{n-1}^{2}$ difference, it is found that

$$
\tilde{d}\left(\rho_{n}\right)=\frac{\left.\min \left(R_{n}^{2}-R_{n-1}^{2}\right)\right|_{n=1, \ldots, N}}{R_{n}^{2}-R_{n-1}^{2}}, \quad \text { for } n=1, \ldots, N,
$$

which is the expression that was used in Section 3.

Some remarks are due with respect to the hereby discussed choice for a (normalized) discrete amplitude density function. Firstly, in view of the correspondence between $\rho_{n}$ and $R_{n}$, it is obvious that $R_{N}$ exceeds $\rho_{N}$. In many cases, the aperture will be construed as the area effectively covered by individual radiators, a choice that allows mapping the beamwidth requirement on a maximum element to center spacing. In that case, $\rho_{N}=R_{\text {ap }}$ and, thus, $R_{N}>R_{\text {ap }}$, as anticipated above. This fact does not conflict with the definition of the density function, the continuous $A(\rho)$ being amenable to extrapolation beyond $R_{\mathrm{ap}}$, while the normalization in (B.6) recalibrates the maximum $\tilde{d}\left(\rho_{n}\right)$ to 1 .

Secondly, the determination of the radii $R_{n}$ according to the condition to yield equal surface integrals of $A(\rho)$ over the relevant annular rings can be easily carried out when the amplitude density is known, as demonstrated in Section 4. However, the handling of the converse situation, when the location of the elements is known and the (equivalent) amplitude density needs being calculated is less evident. To circumvent this difficulty, the radii $R_{n}$ were chosen in Section 3 based on the intrinsic properties of the Fermat spiral, a choice that eventually allowed verifying that the amplitude density function $\tilde{d}\left(\rho_{n}\right)$ is, indeed, constant.

\section{References}

[1] Y. Cailloce, G. Caille, I. Albert, and J. M. Lopez, "A Ka-band direct radiating array providing multiple beams for a satellite multimedia mission," in Proceedings of the IEEE International Symposium on Phased Array Systems and Technology, pp. 403406, Dana Point, Calif, USA, May 2000.

[2] G. Toso, C. Mangenot, and A. G. Roederer, "Sparse and thinned arrays for multiple beam satellite applications," in Proceedings of the 2nd European Conference on Antennas and Propagation (EuCAP'07), pp. 1-4, Edinburgh, UK, November 2007.

[3] M. C. Viganó, G. Toso, S. Selleri, C. Mangenot, P. Angeletti, and G. Pelosi, "GA optimized thinned hexagonal arrays for satellite applications," in Proceedings of the IEEE Antennas and Propagation International Symposium, pp. 3165-3168, Honolulu, Hawaii, USA, June 2007.

[4] G. Caille, Y. Cailloce, C. Guiraud, D. Auroux, T. Touya, and M. Masmousdi, "Large multibeam array antennas with reduced number of active chains," in Proceedings of the 2nd European Conference on Antennas and Propagation (EUCAP '07), pp. 19, Edinburgh, UK, November 2007.

[5] H. Unz, "Linear Arrays with arbitrarily distributed elements," IRE Transactions on Antennas and Propagation, vol. 8, no. 2, pp. 222-223, 1960.

[6] Y. T. Lo and S. W. Lee, "A study of space-tapered arrays," IEEE Transactions on Antennas and Propagation, vol. 14, no. 1, pp. 22-30, 1966.

[7] R. E. Willey, "Space tapering of linear and planar arrays," IEEE Transactions on Antennas and Propagation, vol. 10, no. 4, pp. 369-377, 1962.

[8] R. Harrington, "Sidelobe reduction by nonuniform element spacing," IRE Transactions on Antennas and Propagation, vol. 9, no. 2, pp. 187-192, 1961.

[9] A. Ishimaru, "Theory of unequally-spaced arrays," IRE Transactions on Antennas and Propagation, vol. 10, no. 6, pp. 691702, 1962.

[10] H. Schjaer-Jacobsen and K. Madsen, "Synthesis of nonuniformly spaced arrays using a general nonlinear minimax optimisation method," IEEE Transactions on Antennas and Propagation, vol. 24, no. 4, pp. 501-506, 1976.

[11] W. Doyle, "On approximating linear array factors," Tech. Rep. RM-3530-PR, RAND Corporation, Santa Monica, Calif, USA, February 1963.

[12] T. A. Milligan, "Space-tapered circular (ring) array," IEEE Antennas and Propagation Magazine, vol. 46, no. 3, pp. 70-73, 2004.

[13] M. Vicente-Lozano, F. Ares-Pena, and E. Moreno, "Pencilbeam pattern synthesis with a uniformly excited multi-ring planar antenna," IEEE Antennas and Propagation Magazine, vol. 42, no. 6, pp. 70-74, 2000.

[14] R. L. Haupt, "Optimized element spacing for low sidelobe concentric ring arrays," IEEE Transactions on Antennas and Propagation, vol. 56, no. 1, pp. 266-268, 2008.

[15] G. Toso, M. C. Viganó, and P. Angeletti, "Null-matching for the design of linear aperiodic arrays," in Proceedings of the IEEE Antennas and Propagation International Symposium, pp. 31493152, Honolulu, Hawaii, USA, June 2007.

[16] Y. Chow, "On grating plateaux of nonuniformly spaced arrays," IEEE Transactions on Antennas and Propagation, vol. 13, no. 2, pp. 208-215, 1965. 
[17] M. I. Skolnik, "Nonuniform arrays," in Antenna Theory, Part I, F. J. Collin and R. E. Zucker, Eds., chapter 6, pp. 207-234, McGraw-Hill, New York, NY, USA, 1969.

[18] J. Sahalos, "The orthogonal method of nonuniformly spaced arrays," Proceedings of the IEEE, vol. 62, no. 2, pp. 281-282, 1974.

[19] R. L. Haupt, “Thinned arrays using genetic algorithms," IEEE Transactions on Antennas and Propagation, vol. 42, no. 7, pp. 993-999, 1994.

[20] A. Trucco, "Synthesis of aperiodic planar arrays by a stochastic approach," in Proceedings of the MTS/IEEE Conference (OCEANS '97), vol. 2, pp. 820-825, Halifax, Canada, October 1997.

[21] S. Holm, A. Austeng, K. Iranpour, and J. F. Hopperstad, "Sparse sampling in array processing," in Sampling Theory and Practice, F. Marvasti, Ed., chapter 19, Springer, New York, NY, USA, 2001.

[22] O. M. Bucci, M. D’Urso, T. Isernia, P. Angeletti, and G. Toso, "A new deterministic technique for the design of uniform amplitude sparse arrays," in Proceedings of the 30th ESA Workshop on Antennas for Earth Observation, Science, Telecommunication and Navigation Space Missions, Noordwijk, The Netherlands, May 2008.

[23] D. W. Boeringer, "Phased array including a logarithmic spiral lattice of uniformly spaced radiating and receiving elements," US patent no. 6433754 B1, Silver Spring, Md, USA, April 2002.

[24] T. Taylor, "Design of circular apertures for narrow beamwidth and low sidelobes," IRE Transactions on Antennas and Propagation, vol. 8, no. 1, pp. 17-22, 1960.

[25] J. L. Volakis, Antenna Engineering Handbook, McGraw-Hill, New York, NY, USA, 4th edition, 2007.

[26] A. Okabe, B. Boots, K. Sugihara, and S. N. Chiu, Spatial Tessellations: Concepts and Applications of Voronoi Diagrams, John Wiley \& Sons, Chichester, UK, 2nd edition, 1999.

[27] P. Atela, C. Golé, and S. Hotton, "A dynamical system for plant pattern formation: a rigorous analysis," Journal of Nonlinear Science, vol. 12, no. 6, pp. 641-676, 2002.

[28] R. A. Dunlap, The Golden Ratio and Fibonacci Numbers, World Scientific, Singapore, 1997. 

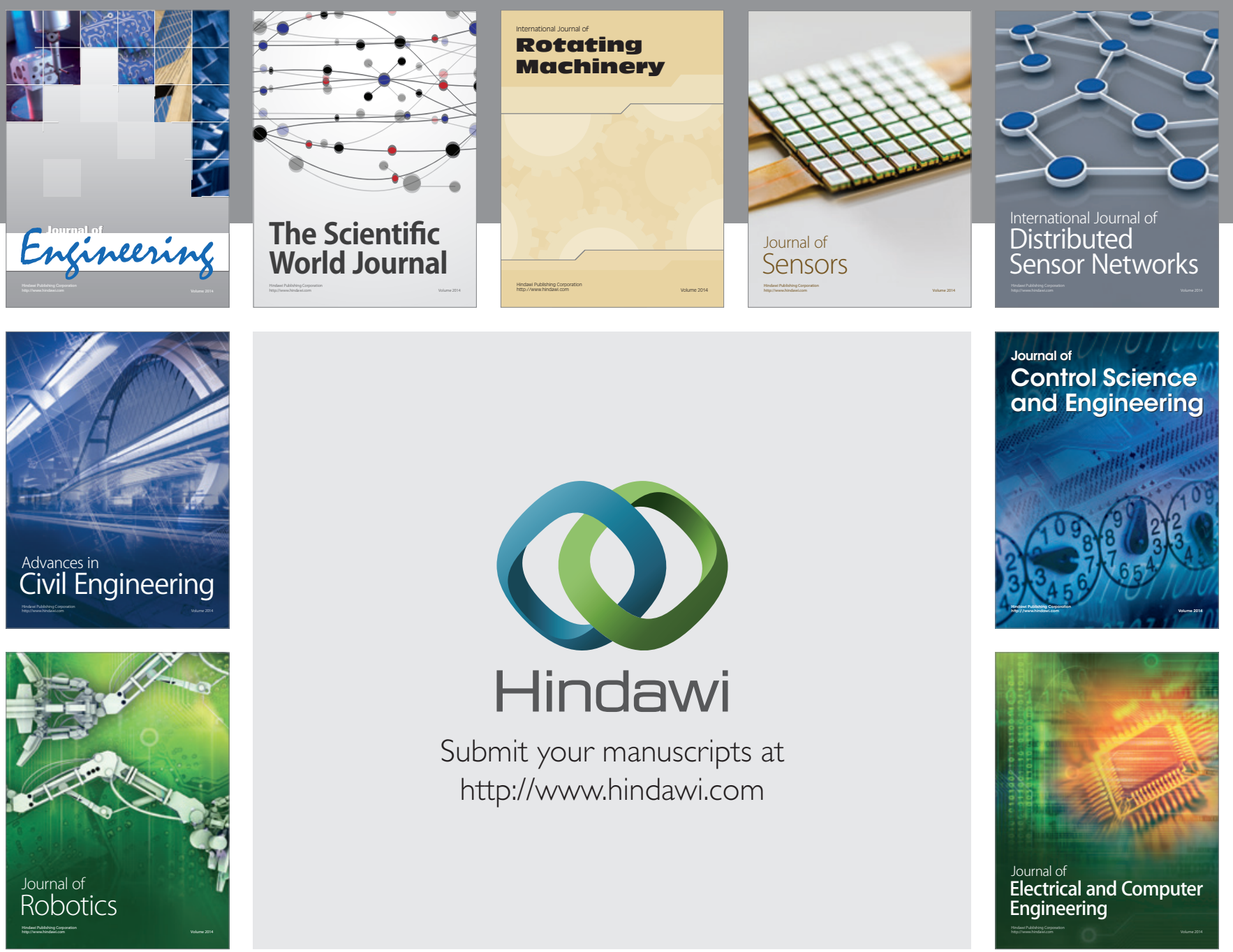

Submit your manuscripts at

http://www.hindawi.com
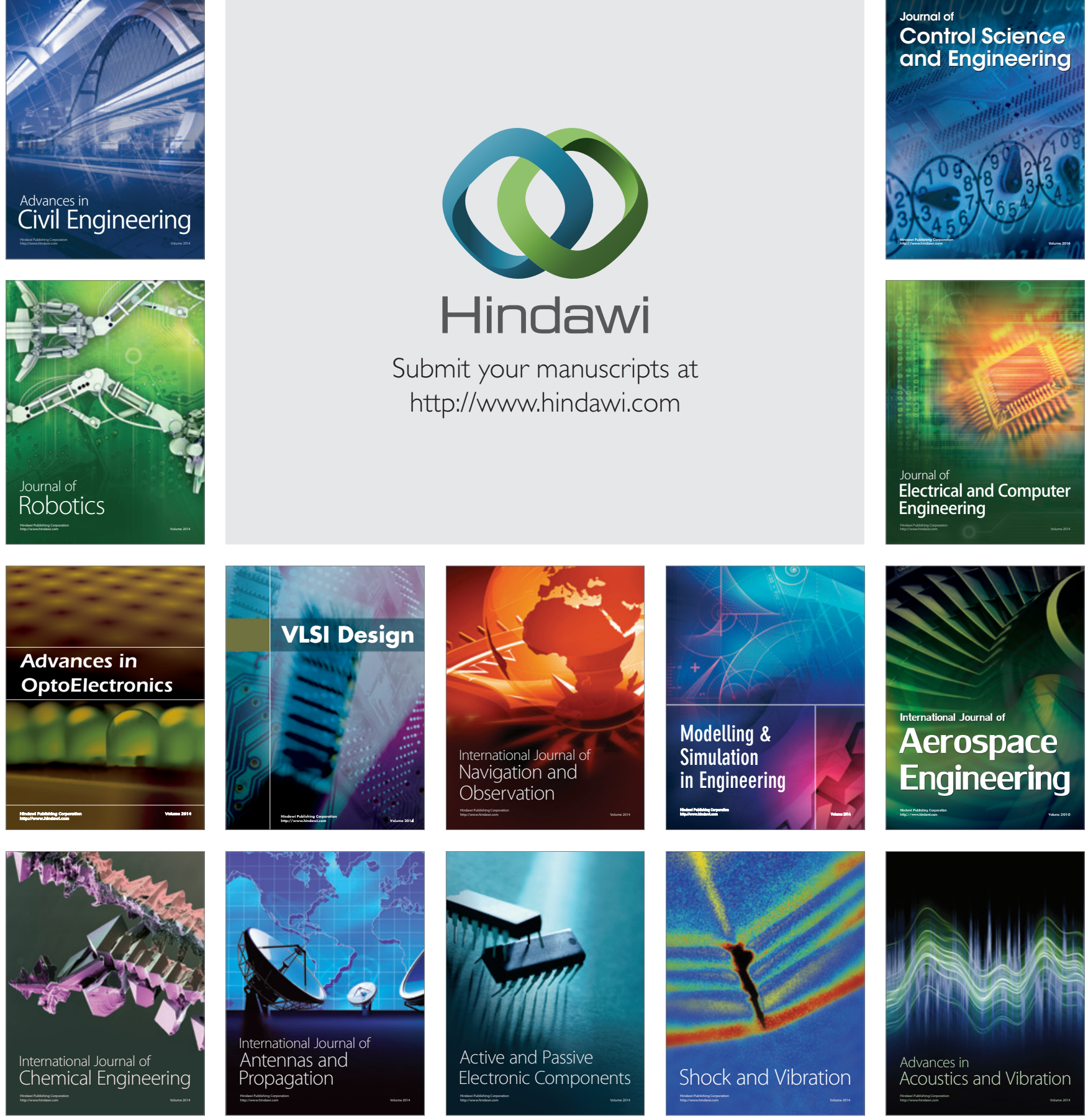